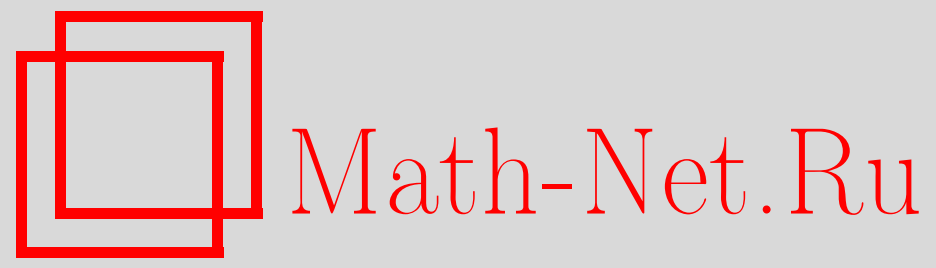

Обцероссийский математический портал 
П. А. Терехин, K вопросу о возмущениях системы Хаара, Матем. заметки, 2004, том 75, выпуск 3, 466-469

DOI: https://doi.org/10.4213/mzm553

Использование Общероссийского математического портала Math-Net.Ru подразумевает, что вы прочитали и согласны с пользовательским соглашением http://www. mathnet.ru/rus/agreement 
Параметры загрузки:

IP : 52.23.180.231

26 апреля 2023 г., 15:34:39

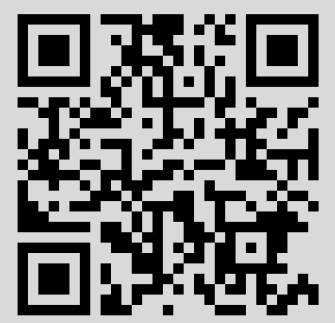




\section{К ВОПРОСУ О ВОЗМУЩЕНИЯХ СИСТЕМЫ ХААРА}

\section{П. А. Терехин}

В статье обсуждаются свойства полноты и базисности в пространстве $L_{p}[0,1], 1 \leqslant p<\infty$, общих систем функций

$$
\left\{1, \varphi_{k, j}\right\}, \quad k=0,1, \ldots, \quad j=0, \ldots, 2^{k}-1
$$

${ }^{2}$ Здесь используется условие $\delta>-\alpha$. В [8] исследованы все случаи, но для наших целей этот представляет наибольший интерес.

Работа выполнена при поддержке Российского фонда фундаментальных исследований, грант № 01-01-00123, программы "Ведушие научные школы РФ", грант НШ-1295.2003.1, и программы INTAS, грант № 99-00089. 
близких в определенном смысле к классической системе функций Хаара

$$
\left\{1, \chi_{k, j}\right\}, \quad k=0,1, \ldots, \quad j=0, \ldots, 2^{k}-1,
$$

где $1=\chi_{[0,1)}$ и $\chi_{k, j}(t)=2^{k / 2} \chi\left(2^{k} t-j\right), \chi=\chi_{[0,1 / 2)}-\chi_{[1 / 2,1)}$. Поводом к такому обсуждению послужил результат работы В.И. Филиппова [1, теорема 3 , с. 600$]$, который мы воспроизводим в эквивалентной формулировке: если $\sum_{k=0}^{\infty} 2^{k / 2} \max _{0 \leqslant j<2^{k}}\left\|\chi_{k, j}-\varphi_{k, j}\right\|_{1}<1$, то система функиий $\left\{1, \varphi_{k, j}\right\}$ полна в пространстве $L_{1}[0,1]$.

В настоящей работе показывается, что этот результат можно усилить, если воспользоваться хорошо известной методикой получения теорем устойчивости представляющих свойств близких систем функций и простейшими свойствами системы Хаара. Именно, справедливы следующие теоремы.

Теорема 1. Eсли $\sum_{k=0}^{\infty} 2^{k / 2} \max _{0 \leqslant j<2^{k}}\left\|\chi_{k, j}-\varphi_{k, j}\right\|_{1}<1$, то система функций $\left\{1, \varphi_{k, j}\right\}$ является базисом пространства $L_{1}[0,1]$.

Аналог сформулированной теоремы верен при любом $p \in[1, \infty)$ :

Tеорема 2. Пусть $1 \leqslant p<\infty, 1 / p+1 / q=1$. Если

$$
\sum_{k=0}^{\infty} 2^{k(1 / 2-1 / q)}\left(\sum_{j=0}^{2^{k}-1}\left\|\chi_{k, j}-\varphi_{k, j}\right\|_{p}^{q}\right)^{1 / q}<1,
$$

то система функиий $\left\{1, \varphi_{k, j}\right\}$ является базисом пространства $L_{p}[0,1]$.

Теорема 2 допускает дальнейшее усиление.

Tеорема 3. Пусть $1 \leqslant p<\infty, 1 / p+1 / q=1$. Если система функций $\left\{1, \varphi_{k, j}\right\} \omega-л u$ нейно независима в $L_{p}[0,1] и$

$$
\sum_{k=0}^{\infty} 2^{k(1 / 2-1 / q)}\left(\sum_{j=0}^{2^{k}-1}\left\|\chi_{k, j}-\varphi_{k, j}\right\|_{p}^{q}\right)^{1 / q}<\infty
$$

то система функиий $\left\{1, \varphi_{k, j}\right\}$ является базисом пространства $L_{p}[0,1]$.

Напомним, что система функций $\left\{1, \varphi_{k, j}\right\}$ называется $\omega$-линейно независимой в пространстве $L_{p}[0,1]$, если из сходимости в этом пространстве нуль-ряда

$$
c_{-1} 1+\sum_{k=0}^{\infty} \sum_{j=0}^{2^{k}-1} c_{k, j} \varphi_{k, j}=0
$$

следует равенство нулю всех его коэффициентов

$$
c_{-1}=0, \quad c_{k, j}=0, \quad k=0,1, \ldots, \quad j=0, \ldots, 2^{k}-1 .
$$

ДОКАЗАТЕЛЬСТВО ТЕОРЕМЫ 3. Пусть

$$
f=(f, 1) 1+\sum_{k=0}^{\infty} \sum_{j=0}^{2^{k}-1}\left(f, \chi_{k, j}\right) \chi_{k, j}
$$

- ряд Фурье-Хаара функции $f \in L_{p}[0,1]$. Определим конечномерные операторы $K_{n}: L_{p}[0,1]$ $\rightarrow L_{p}[0,1], n=0,1, \ldots$, равенством

$$
K_{n} f=\sum_{k=0}^{n} \sum_{j=0}^{2^{k}-1}\left(f, \chi_{k, j}\right)\left(\chi_{k, j}-\varphi_{k, j}\right) .
$$


Нетрудно видеть, что сопряженные операторы $K_{n}^{*}: L_{q}[0,1] \rightarrow L_{q}[0,1]$ даются равенством

$$
K_{n}^{*} g=\sum_{k=0}^{n} \sum_{j=0}^{2^{k}-1}\left(g, \chi_{k, j}-\varphi_{k, j}\right) \chi_{k, j}
$$

Имеем

$$
\begin{aligned}
\left\|K_{n+p}^{*} g-K_{n}^{*} g\right\|_{q} & \leqslant \sum_{k=n+1}^{n+p}\left\|\sum_{j=0}^{2^{k}-1}\left(g, \chi_{k, j}-\varphi_{k, j}\right) \chi_{k, j}\right\|_{q} \\
& =\sum_{k=n+1}^{n+p} 2^{k(1 / 2-1 / q)}\left(\sum_{j=0}^{2^{k}-1}\left|\left(g, \chi_{k, j}-\varphi_{k, j}\right)\right|^{q}\right)^{1 / q} \\
& \leqslant\|g\|_{q} \sum_{k=n+1}^{n+p} 2^{k(1 / 2-1 / q)}\left(\sum_{j=0}^{2^{k}-1}\left\|\chi_{k, j}-\varphi_{k, j}\right\|_{p}^{q}\right)^{1 / q} .
\end{aligned}
$$

Отсюда и в силу условий теоремы последовательность $K_{n}^{*}$ сходится по равномерной операторной норме. То же справедливо и для последовательности $K_{n}$. Таким образом, равенство

$$
K f=\sum_{k=0}^{\infty} \sum_{j=0}^{2^{k}-1}\left(f, \chi_{k, j}\right)\left(\chi_{k, j}-\varphi_{k, j}\right)
$$

определяет компактный оператор в $L_{p}[0,1]$. Далее, в силу условия $\omega$-линейной независимости системы функций $\left\{1, \varphi_{k, j}\right\}$ единица не является собственным значением оператора $K$. Поэтому оператор $A=I-K$ обратим. Последнее утверждение основано на следующем хорошо известном факте, непосредственно вытекающем из альтернативы Фредгольма: если число $\lambda \neq 0$ не является собственным значением компактного оператора $K$, то оператор $\lambda I-K$ обратим (см., например, [2, с. 87]). Осталось заметить, что $A 1=1, A \chi_{k, j}=\varphi_{k, j}, k=0,1, \ldots, j=0, \ldots, 2^{k}-1$, т.е. система $\left\{1, \varphi_{k, j}\right\}$ эквивалентна системе Хаара и потому является базисом.

ДоКАЗАТЕЛЬСтво тЕОРЕмы 2. Так же, как и при доказательстве теоремы 3 , получим оценку

$$
\left\|K_{n}^{*} g\right\|_{q} \leqslant\|g\|_{q} \sum_{k=0}^{n} 2^{k(1 / 2-1 / q)}\left(\sum_{j=0}^{2^{k}-1}\left\|\chi_{k, j}-\varphi_{k, j}\right\|_{p}^{q}\right)^{1 / q}
$$

откуда

$$
\|K\| \leqslant \sum_{k=0}^{\infty} 2^{k(1 / 2-1 / q)}\left(\sum_{j=0}^{2^{k}-1}\left\|\chi_{k, j}-\varphi_{k, j}\right\|_{p}^{q}\right)^{1 / q}<1
$$

и оператор $A=I-K$ снова обратим.

ДоКАЗАТЕЛЬСТво тЕОРЕмЫ 1 . Достаточно положить в теореме $2 p=1$ и заметить, что при $q=\infty$ формально имеем

$$
2^{k(1 / 2-1 / q)}\left(\sum_{j=0}^{2^{k}-1}\left\|\chi_{k, j}-\varphi_{k, j}\right\|_{p}^{q}\right)^{1 / q}=2^{k / 2} \max _{0 \leqslant j<2^{k}}\left\|\chi_{k, j}-\varphi_{k, j}\right\|_{1} .
$$

Применим полученные резултаты к вопросу о базисности семейств нестационарных всплесков. Всюду далее функции $\varphi_{k}(t), t \in R, k=0,1, \ldots$, имеют носитель $\operatorname{supp} \varphi_{k} \subset[0,1]$ и $\varphi_{k} \in L_{p}[0,1]$, $1 \leqslant p<\infty$. Семейство функций

$$
\varphi_{k, j}(t)=2^{k / 2} \varphi_{k}\left(2^{k} t-j\right), \quad k=0,1, \ldots, \quad j=0, \ldots, 2^{k}-1,
$$


назьвается нестационарным всплеском (в стационарном случае $\varphi_{k}=\varphi$ для всех $k=0,1, \ldots$ ).

Предположим, далее, что функции $\varphi_{k}$ имеют нулевой интеграл:

$$
\left(\varphi_{k}, 1\right)=\int_{0}^{1} \varphi_{k}(t) d t=0
$$

и нормированы условием

$$
\left(\varphi_{k}, \chi\right)=\int_{0}^{1 / 2} \varphi_{k}(t) d t-\int_{1 / 2}^{1} \varphi_{k}(t) d t=1
$$

Лемма 1. Пусть $m \leqslant k, 0 \leqslant j<2^{k}, 0 \leqslant i<2^{m}$. Тогдa $\left(\varphi_{k, j}, \chi_{m, i}\right)=\delta_{m k} \delta_{i j}$, где $\delta-$ символ Кронекера.

ДоКАЗАТЕльСтво дословно повторяет рассуждения, устанавливающие формулы $\left(2.5_{2}\right)$ и $\left(2.5_{3}\right)$ предложения 4 работы автора [2], надо лишш учесть нормировку (2) и нестационарность всплеска, которая в данном случае не оказьвает влияния на доказательство.

ЛЕмма 2. Система функиий $\left\{1, \varphi_{k, j}\right\}$ является $\omega$-линейно независимой в пространcmв е $L_{p}[0,1]$.

ДокаЗАТЕЛЬСтво. Пусть $c_{-1} 1+\sum_{k=0}^{\infty} \sum_{j=0}^{2^{k}-1} c_{k, j} \varphi_{k, j}=0$. Умножая это равенство скалярно на функцию 1 и учитьвая равенство (1), получаем $c_{-1}=0$. Затем, умножая скалярно на функцию $\chi$ и применяя лемму 1 с $m=0$, получаем $c_{0,0}=0$. Предположим, что для всех $k<m$ равенство $c_{k, j}=0$ доказано при всех $j=0, \ldots, 2^{k}-1$. Тогда $\sum_{k=m}^{\infty} \sum_{j=0}^{2^{k}-1} c_{k, j} \varphi_{k, j}=0$. Умножая это равенство на функцию $\chi_{m, i}$ и применяя лемму 1 , получаем $c_{m, i}=0, i=0, \ldots, 2^{m}-1$. По индукции заключаем, что все коэффициенты исходного нуль-ряда равны нулю.

ТЕОРема 4. Пусть функиии $\varphi_{k}, k=0,1, \ldots$, удовлетворяют условиям (1), (2) и условию

$$
\sum_{k=0}^{\infty} 2^{k(1-1 / p)}\left\|\chi-\varphi_{k}\right\|_{p}<\infty
$$

Тогда нестационарный всплеск $\left\{1, \varphi_{k, j}\right\}$ является базисом пространства $L_{p}[0,1]$.

ДоКАЗАТЕЛЬСтво непосредственно следует из теоремы 3 и леммы 2 с учетом равенства

$$
\sum_{k=0}^{\infty} 2^{k(1 / 2-1 / q)}\left(\sum_{j=0}^{2^{k}-1}\left\|\chi_{k, j}-\varphi_{k, j}\right\|_{p}^{q}\right)^{1 / q}=\sum_{k=0}^{\infty} 2^{k(1-1 / p)}\left\|\chi-\varphi_{k}\right\|_{p}
$$

ЗАмЕч АниЕ. 1. К случаю стационарного всплеска теорема 4 не применима. Условия базисности стационарных всплесков в пространстве $L_{2}[0,1]$ даны в работе [3].

2. Можно показать, что в условиях леммы 2 нестационарный всплеск $\left\{1, \varphi_{k, j}\right\}$ не только $\omega$-линейно независим, но и минимален в пространстве $L_{p}[0,1]$. 


\section{СПИСОК ЦИТИРОВАННОЙ ЛИТЕРАТУРЫ}

1. Филиппов В. И. // Матем. заметки. 1999. Т. 66. №4. С. 596-602. 2. Кириллов А. А., Гвишиани А. Д. Теоремы и задачи функционаљього анализа. М.: Наука, 1979. 3. Терехин П.А. // Матем. заметки. 2002. Т. 72. № 4. С. 547-560.

Саратовский государственный университет им. Н. Г. Черньшшевского 